\title{
OPTIMIZATION IN ABSTRACT SPACES
}

\author{
LESLIE IAN TRUDZIK
}

The thesis is concerned with the abstract mathematical programming problem, namely, the problem of minimizing a function of an abstract variable subject to some constraint on the variable. A number of recent developments in finite and semi-infinite programing theory are extended to the abstract case, some under weaker hypotheses than previously known. At the same time new optimality criteria are obtained, generated directly from the abstract problem.

In Chapter 1 the continuity of cone-convex functions is considered in parallel to the classical theory for scalar-valued functions. Various characterizations of scalar-valued semi-continuity are extended to and compared in the abstract case. While upper semi-continuity characterizes continuity of cone-convex functions, a new form of lower semi-continuity (which we call lower quasi-continuity) turns out to be sufficient for continuity of cone-convex functions. As an important preliminary it is proved that cone-convex functions with finite dimensional domain share, with their scalar predecessors, the property of being continuous along lines (or finite dimensional subspaces) inside their effective domains.

In Chapter 2 optimality for the abstract mathematical program is characterized by restricted Kuhn-Tucker conditions. Although the general form of these conditions is well known, a simpler and more general proof is given for the existence of restricted slater points, and hence restricted

Received 9 December 1983. Thesis submitted to the University of Melbourne, May 1983. Degree approved October 1983. Supervisor: Dr B.D. Craven.

Copyright Clearance Centre, Inc. Serial-fee code: 0004-9727/84 $\$$ A2.00 +0.00 . 
Kuhn-Tucker conditions. The conditions depend upon knowledge of the minimal set of binding constraints and an iterative procedure for the evaluation of this set is supplied. Importantly, the procedure cannot jam short of its objective.

In Chapter 3 the gap between the Kuhn-Tucker (sufficient) conditions and the Fritz-John (necessary) conditions is bridged by the introduction of doubly asymptotic Kuhn-Tucker conditions. The conditions involve asymptoticity in both the adjoint equation and the complementary slackness condition. Under mild assumptions the conditions characterize optimality and furnish asymptotic duality results. In particular they characterize optimality for all linear programs. Constraint qualifications which permit one or both of the asymptotic operations to be omitted are also discussed. A new constraint qualification, based on the Farkas-Minkowski constraint qualification in semi-infinite programming, is introduced.

Chapter 4 considers programs with constraints satisfying certain "mean value" and "uniformly decreasing" properties. These are quite distinct from the usual form of constraint qualification and have already appeared in the literature in the context of semi-infinite programming. They permit extensions of and unify a wide range of feasible direction optimality criteria known for finite programming. Optimality is characterized by the inconsistency of systems of cone inclusions, with associated Lagrange multiplier results, and by the solutions to associated simpler programs. The latter lead to an iterative procedure for checking the optimality of feasible points.

Chapter 5 is concerned with the stability of perturbed convex programs in reflexive Banach spaces. Both the feasible set and the objective function are subject to perturbation, and stability is considered for each of the optimal set, the program value, the feasible set and the Lagrange multiplier set. The emphasis is on the determination of stable regions within the parameter space. In particular the set of parameters for which the optimal set is weak compact and non-empty is shown to be topologically open. This extends a well known result in finite programing. The restricted Kuhn-Tucker theory of Chapter 2 is used to define regions of stability without requiring the usual constraint qualification or regularity assumption. The results are also applied to the problem of 
approximating a given (possibly ill-posed) program by well-posed programs.

\section{Mi I Iward Street,}

Brunswick, Victoria 3056,

Australia. 